\title{
Spectrophotometric detection of Pigments from Aspergillus and Penicillium isolates
}

\author{
B. N. Narendrababu, S. Shishupala* \\ Department of Microbiology, Davangere University, Shivagangotri campus, Davangere - 577002.Karnataka, India.
}

\begin{tabular}{|c|c|}
\hline ARTICLE INFO & ABSTRACT \\
\hline $\begin{array}{l}\text { Article history: } \\
\text { Received on: } 03 / 08 / 2016 \\
\text { Revised on: } 13 / 09 / 2016 \\
\text { Accepted on: } 05 / 10 / 2016 \\
\text { Available online: } 20 / 01 / 2017\end{array}$ & $\begin{array}{l}\text { Fungal pigments are extremely important for biotechnological exploitation. The present study was conducted to } \\
\text { detect and analyze the pigments produced by selected Aspergillus and Penicillium isolates. The pigment } \\
\text { producing fungi were isolated from soil and air. Fungi producing yellow and orange-red pigments were cultured } \\
\text { on Potato dextrose broth and Czapek-Dox broth separately. Cellular pigments were extracted from mycelial mass } \\
\text { and secretory pigments were extracted from culture filtrates using chloroform and ethyl acetate separately. Visible }\end{array}$ \\
\hline $\begin{array}{l}\text { Key words: } \\
\text { Aspergillus, Penicillium, } \\
\text { Pigments, } \\
\text { Spectrophotometry. }\end{array}$ & $\begin{array}{l}\text { pigments were observed mostly in mycelial mass extracted in ethyl acetate. UV-Visible spectroscopic analysis } \\
\text { provided detection of yellow pigment }(380 \mathrm{~nm}) \text { in Aspergillus isolate and orange-red pigment }(463 \mathrm{~nm}) \text { in } \\
\text { Penicillium sp. The results also indicated that, the production of pigments in these fungi is dependent on the } \\
\text { availability of nutrients in the medium. Extractability of the pigments was dependent on the solvent. These fungi } \\
\text { may be exploited biotechnologically by maintaining suitable culture conditions for industrial production of } \\
\text { pigments. }\end{array}$ \\
\hline
\end{tabular}

\section{INTRODUCTION}

Biological pigments are of significance with different roles. Various types of algae and bacteria are known to produce several pigments apart from plants. Many of them are protective in function. Pigments of biological origin are also being exploited for commercial purpose. Some of the plant pigments are being used as food colourants and also for dyeing fabrics. In the past decade, interest on natural pigments is increasing due to ill effects of synthetic colourants. Colours provide attractive appearance to marketable products in food, textiles and pharmaceutical industries. Interest on pigments from natural sources has increased due to the toxicity problems caused by the synthetic pigments. In this way, the pigments from microbial sources are a good alternative. To counter the hazardous effects of synthetic colourants, there is worldwide interest in process development for the production of pigments from natural sources [1, 2, 3, 4]. Plants and microorganisms are the two major sources of natural pigments. The microbial pigments are of great interest owing to the stability of the pigments produced and the availability of cultivation technology [1]. Pigments like carotenoids, anthraquinone and

\footnotetext{
* Corresponding Author

Shantharaju Shishupala, Department of Microbiology, Davangere University, Shivagangotri campus, Davangere - 577002. Karnataka, India.Email: ssdumb @ gmail.com
}

chlorophyll have been produced from yeast, fungi, bacteria and algae [5,6,7]. Significant metabolites of fungal origin are important commercially. Biotechnological applications of fungal metabolites are mostly confined to enzymes and antibiotics. However, various types of fungal pigments may be exploited in biotechnology industry. Common fungal pigments detected belong to the group of melanins, carotenoids, lycopene and xanthophylls $[8,9,10,11]$. These pigments offer protection to fungi and show diverse biological activity [12, 13]. With globalization, there is an increasing trend for products with natural and safe pigments. Industrial applications of fungal pigments may pave the way for alternative source of colourants in biotechnology [6, 14]. Hence, in this paper we report the detection and analysis of pigments from selected fungi.

\section{MATERIALS AND METHODS}

\subsection{Isolation of pigment producing fungi}

Sterilized Potato dextrose agar (PDA) plates were exposed to air for $10 \mathrm{~min}$ at different areas in Davangere University campus. Soil samples were collected randomly in Davangere University and soil suspension was spread plated on PDA. The plates were incubated at room temperature up to five days. Different fungal isolates appearing on PDA plates having pigments were selected and transferred onto PDA slants to get pure cultures. The pigment producing fungi were identified using fungal identification manual $[15,16]$. 


\subsection{Fungal cultures for pigment production}

Two loopful of spores from five selected fungi were separately inoculated into $30 \mathrm{ml}$ of Potato dextrose broth (PDB) and to $30 \mathrm{ml}$ of Czapek-Dox broth (CDB) taken in $100 \mathrm{ml}$ conical flasks aseptically. These cultures were incubated under stationary condition at room temperature $\left(28 \pm 4^{0} \mathrm{C}\right)$ for seven days for pigment production. The uninoculated $\mathrm{PDB}$ and CDB were used as control.

\subsection{Extraction of cellular and secretory pigments from fungi}

The mycelial mass obtained from each culture broth was separated by filtration and soaked overnight in chloroform and ethyl acetate separately. The mycelium was crushed using glass rod and shaken well. The cellular debris were removed and the supernatant containing soluble compounds were extracted with the same solvent in a separating funnel. The solvent fraction was separated and evaporated to dryness at room temperature. The extract was observed for colour of the pigment.

The culture filtrate of individual fungus was obtained by filtering through ordinary filter paper. The compounds soluble in ethyl acetate and chloroform were extracted separately. The culture filtrate and solvent were taken in a separating funnel (1:1, $\mathrm{v} / \mathrm{v})$ and mixed well. The solvent fraction was separated and concentrated by evaporation. The extracts were re-dissolved in minimum amount of respective solvent and observed for colour. Only media extracted in respective solvent served as controls.

\subsection{UV-Visible spectrophotometry of fungal extracts}

The chloroform and ethyl acetate soluble compounds from mycelial mass and culture filtrates of different fungal isolates were subjected to UV-Visible spectrophotometry (ELICO, SL 159 UV-VIS Spectrophotometer). The absorption maxima of compounds present in chloroform and ethyl acetate extracts were determined by subjecting the samples to wavelength scan from $200 \mathrm{~nm}$ to $800 \mathrm{~nm}$. One hundred microlitre of each sample in two milliliter of respective solvent was used by keeping specific solvent as reference blank. The PDB and CDB media extracted with the solvents were used as controls.

\section{RESULTS AND DISCUSSION}

\subsection{Isolation of pigment producing fungi}

Out of many fungi found in soil and air, only five isolates were found to produce pigments. These fungi belonged to Penicillium and Aspergillus species. Out of the selected fungi three isolates belonging to Penicillium sp. were given with culture collection number DUMB11, DUMB12 and DUMB15. Two isolates belonged to Aspergillus sp. and designated as DUMB13 and DUMB14. Penicillium sp. (DUMB12) and (DUMB15) and Aspergillus sp. (DUMB14) were isolated from soil. Penicillium sp. (DUMB11) and Aspergillus sp. (DUMB13) were isolated from air. The pure cultures of these fungi were observed to have both cellular and secretory pigments (Fig. 1). Both Penicillium sp. and Aspergillus sp. have been found to produce different kinds of pigments [9, 17]. Hence, selection of fungal isolates was found to be appropriate for pigment detection and analysis.

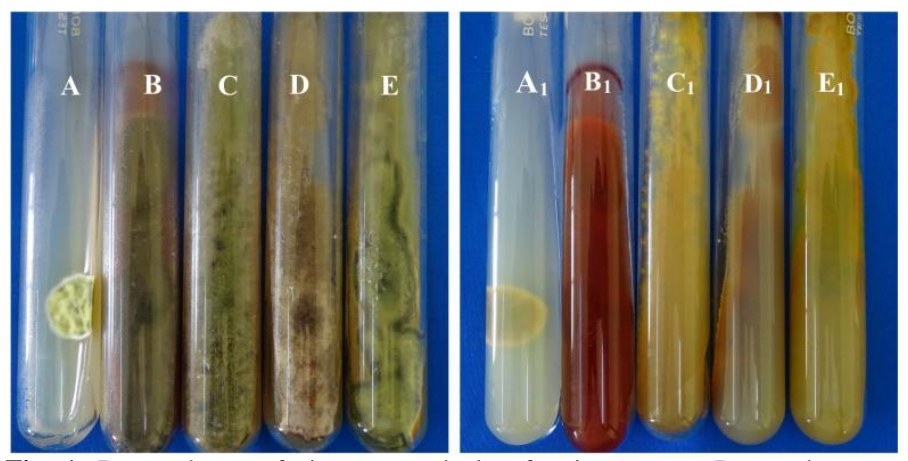

Fig. 1: Pure cultures of pigment producing fungi grown on Potato dextrose agar slants.

A-Penicillium sp. (DUMB 11), B-Penicillium sp. (DUMB 12), CAspergillus sp. (DUMB 13), D- Aspergillus sp. (DUMB 14), EPenicillium sp. (DUMB 15).

$A_{1} B_{1} C_{1} D_{1} E_{1}=$ Reverse view of respective fungal culture slants.

\subsection{Fungal cultures for pigment production}

Highly appreciative growth of selected fungi were noticed in PDB when compared to CDB. At the same time, intense pigments were observed in the fungal cultures grown in PDB. The solvents chloroform and ethyl acetate extracts also showed pigments.

\subsection{Extraction of cellular and secretory pigments from fungi}

From the mycelial mass, cellular pigments were extracted separately in two solvents. Interesting range of pigments were extracted in fungal samples tested. In PDB Penicillium isolates DUMB11 and DUMB12 showed light yellow coloured compounds as cellular pigments soluble in chloroform. The other Penicillium isolate DUMB15 showed orange-red pigment in the same solvent. Aspergillus isolates DUMB13 and DUMB14 grown in PDB showed red and yellow cellular pigments soluble in chloroform respectively. However, among the five fungal isolates grown in CDB, only Aspergillus sp. DUMB14 showed dark yellow cellular pigment soluble in chloroform. From the fungal culture filtrates, only Aspergillus sp. DUMB14 showed chloroform soluble yellow pigment being secreted to PDB. All other fungi did not show chloroform soluble pigments secreted into the medium. The nutrients and culture conditions greatly influence pigment production in fungi $[18,19]$. In case of ethyl acetate as solvent, Penicillium isolate DUMB11 showed light yellow cellular pigment when grown in PDB. The other two Penicillium isolates DUMB12 and DUMB15 showed orange-red cellular pigment when extracted with ethyl acetate. The Aspergillus isolates DUMB13 and DUMB14 showed cellular pigment orange and brown respectively when grown in PDB and extracted with ethyl acetate. Cellular pigments from Aspergillus DUMB13 were yellow and DUMB14 was light red when grown on CDB. Penicillium DUMB12 showed ethyl acetate soluble light red secretory pigment in PDB. Aspergillus sp. DUMB14 and Penicillium DUMB15 showed secreted yellow pigment in PDB extracted with ethyl acetate. None 
of the isolates were found to show secretory pigments soluble in ethyl acetate when grown on CDB. Natural media appears to provide suitable substrates for pigment production in fungi $[5,19]$. In the present study also more pigments were detected in fungi grown on PDB rather than CDB. This clearly demonstrates production of pigments in Penicillium and Aspergillus is possible on natural media ingredients. Differential solubility of the fungal pigments in chloroform and ethyl acetate was also evident. This indicates ability of fungi to produce various pigments soluble in different solvents. The results also showed cellular pigments were more when compared to secretory pigments. Production of red pigment by Penicillium soluble in ethyl acetate has been reported [17]. Our results are in confirmation with this study, showing Penicillium isolates DUMB12 and DUMB15 producing orangered pigment soluble in ethyl acetate. Even in case of Aspergillus nidulans, dark brown melanin pigments are produced in the mycelium [9]. In our study, the Aspergillus isolate DUMB14 produced brown pigment soluble in ethyl acetate. Detection of different types of pigments produced by fungi was possible showing differential solubility.

\subsection{UV-Visible spectrophotometry of fungal extracts}

The results of UV-Visible spectroscopy revealed the presence of different compounds showing various peaks based on absorption maxima. Only PDB extracted in chloroform showed absorption peaks at wavelength $235 \mathrm{~nm}, 246 \mathrm{~nm}$ and $271 \mathrm{~nm}$. In ethyl acetate extracts of PDB absorption peaks were found in the range of $248 \mathrm{~nm}$ and $278 \mathrm{~nm}$. Likewise, only CDB extracted in chloroform showed peaks in the range of $266 \mathrm{~nm}$ to $280 \mathrm{~nm}$ where as ethyl acetate extract of CDB showed few peaks between 230 to $280 \mathrm{~nm}$. All these peaks do not indicate the presence of pigments. Only substances absorbing visible light (400 to $700 \mathrm{~nm}$ ) appears to have colour [20]. In case of Penicillium sp. (DUMB 11) mycelial mass extracted in chloroform showed peak at 233 and $257 \mathrm{~nm}$ when grown on both the media tested (Fig. 2).

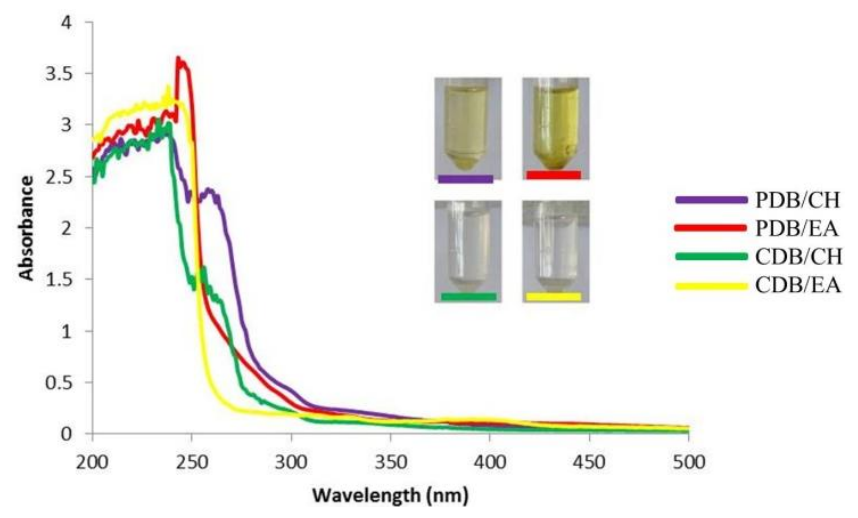

Fig. 2: UV-Visible spectra of mycelial mass of Penicillium sp. (DUMB 11) grown in different media extracted with different solvents. PDB-Potato dextrose broth; CDB-Czapek-Dox broth; CH-Chloroform; EA- Ethyl acetate. Inset- Solvent extracts used for analysis.

However, slight yellow colour was visible in PDB cultured mycelial mass extracted in ethyl acetate, peaks were observed in the range of $240 \mathrm{~nm}$. In case of secretory compounds Penicillium sp. (DUMB 11) showed no significant peaks nor major colour (Fig. 3).

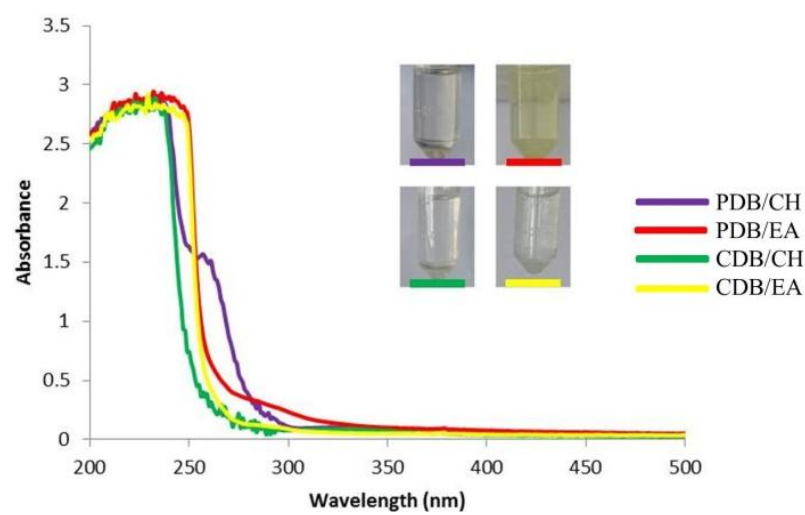

Fig. 3: UV-Visible spectra of culture filtrate of Penicillium sp. (DUMB 11) grown in different media extracted with different solvents. PDB-Potato dextrose broth; CDB-Czapek-Dox broth; CH-Chloroform; EA- Ethyl acetate. Inset- Solvent extracts used for analysis.

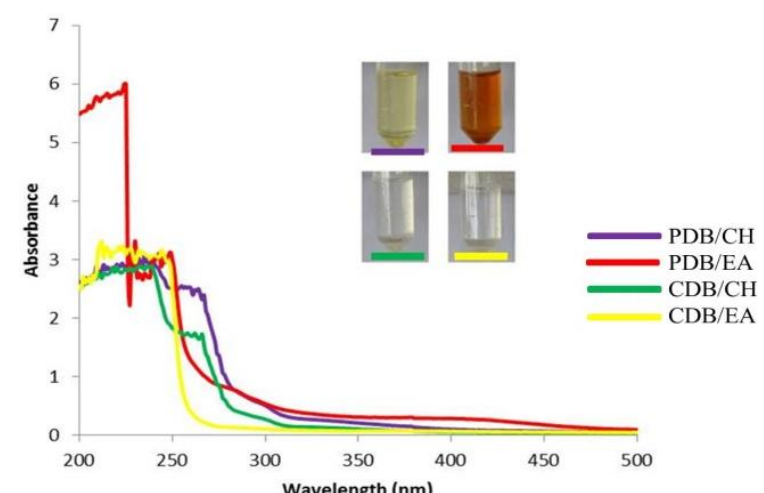

Fig. 4: UV-Visible spectra of mycelial mass of Penicillium sp. (DUMB 12) grown in different media extracted with different solvents. PDB-Potato dextrose broth; CDB-Czapek-Dox broth; $\mathrm{CH}$-Chloroform; EA- Ethyl acetate. Inset- Solvent extracts used for analysis.

The absorption spectra from mycelial mass of Penicillium sp. (DUMB 12) is showed in Fig. 4. Threemajor peaks from the range of $225 \mathrm{~nm}$ to $265 \mathrm{~nm}$ were obtained. A peak at 225 $\mathrm{nm}$ was unique to ethyl acetate extract of mycelial mass from Penicillium sp. (DUMB 12) grown on PDB. However, the secretory pigment did not show significant peaks in the visible range in spite of colour observed in ethyl acetate extract of culture filtrate (Fig. 5). In case of Aspergillus sp. (DUMB 13), a significant peak at $453 \mathrm{~nm}$ was observed in ethyl acetate extracts of mycelial mass grown on PDB (Fig. 6). The same sample showed orange colour in visual observation. Absorption maxima of $450 \mathrm{~nm}$ corresponds to orange pigment [20]. The present result clearly indicated observation of orange colour and detection of the compound by spectrophotometry. Species of Aspergillus are known to produce different coloured pigments including melanins [9]. Probably, the strains tested here could produce related pigments. In the culture filtrate of Aspergillus sp. (DUMB 13) no significant pigments were detected (Fig. 7). The fungus 
Aspergillus sp. (DUMB 14) showed ranges of pigments. The mycelial mass grown in CDB showed detectable yellow pigment corresponding to $380 \mathrm{~nm}$ of absorption (Fig. 8).

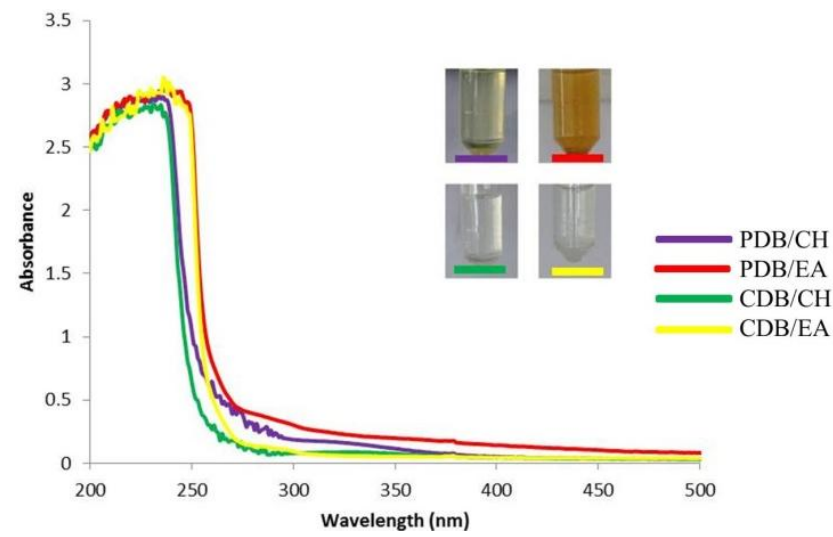

Fig. 5: UV-Visible spectra of culture filtrate of Penicillium sp. (DUMB 12) grown in different media extracted with different solvents. PDB-Potato dextrose broth; CDB-Czapek-Dox broth; CH-Chloroform; EA- Ethyl acetate. Inset- Solvent extracts used for analysis.

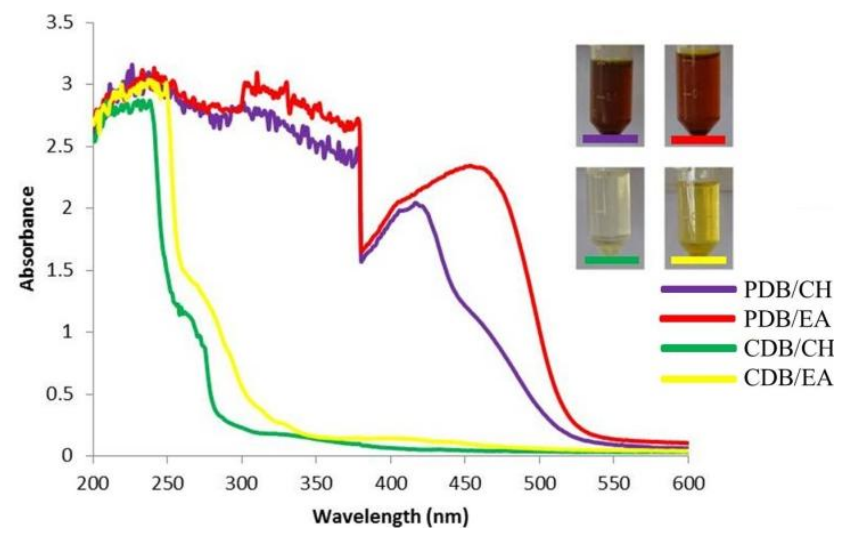

Fig. 6: UV-Visible spectra of mycelial mass of Aspergillus sp. (DUMB 13) grown in different media extracted with different solvents; PDB-Potato dextrose broth; CDB-Czapek-Dox broth; $\mathrm{CH}-\mathrm{Chloroform}$; EA- Ethyl acetate. Inset- Solvent extracts used for analysis.

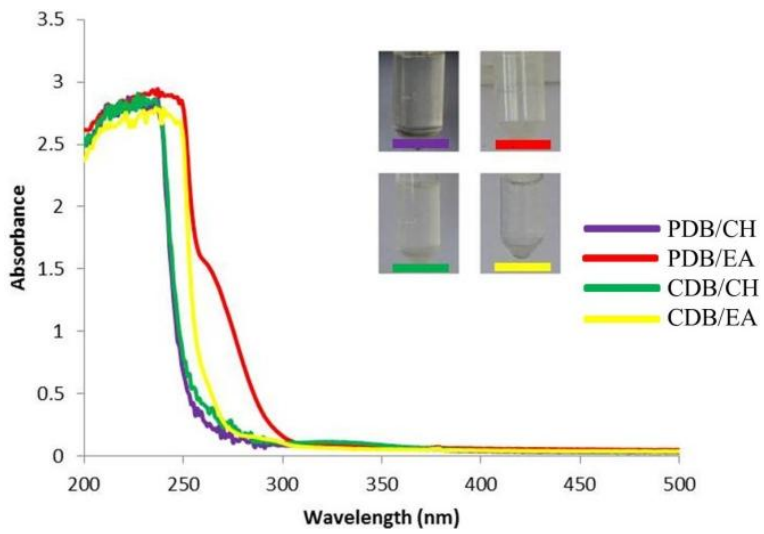

Fig. 7: UV-Visible spectra of culture filtrate of Aspergillus sp. (DUMB 13) grown in different media extracted with different solvents. PDB-Potato dextrose broth; CDB-Czapek-Dox broth; CH-Chloroform; EA- Ethyl acetate. Inset- Solvent extracts used for analysis.

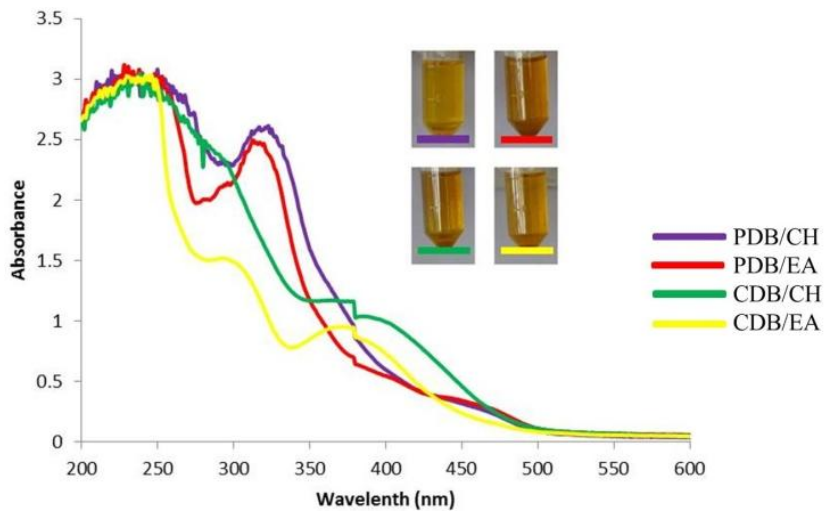

Fig. 8: UV-Visible spectra of mycelial mass of Aspergillus sp. (DUMB 14) grown in different media extracted with different solvents. PDB-Potato dextrose broth; CDB-Czapek-Dox broth; $\mathrm{CH}$-Chloroform; EA- Ethyl acetate. Inset- Solvent extracts used for analysis.

UV-Visible absorption spectra has been used as method to detect the fungal pigments [10, 21]. In case of Monascus purpureus pigments, the absorption peak at $390 \mathrm{~nm}$ indicated yellow pigment and peak at $500 \mathrm{~nm}$ indicated a red pigment [22]. In our study, the major peak corresponds to $240 \mathrm{~nm}$ to $320 \mathrm{~nm}$ from mycelial mass grown in PDB was not corresponding to any pigment but indicated effect of nutrients on metabolite production by fungi. The culture filtrate of this fungus did not show any significant pigment peaks (Fig. 9). The pigment hypocrellin production by Shiraia bambusicola, based on nutrient sources was also reported [19].

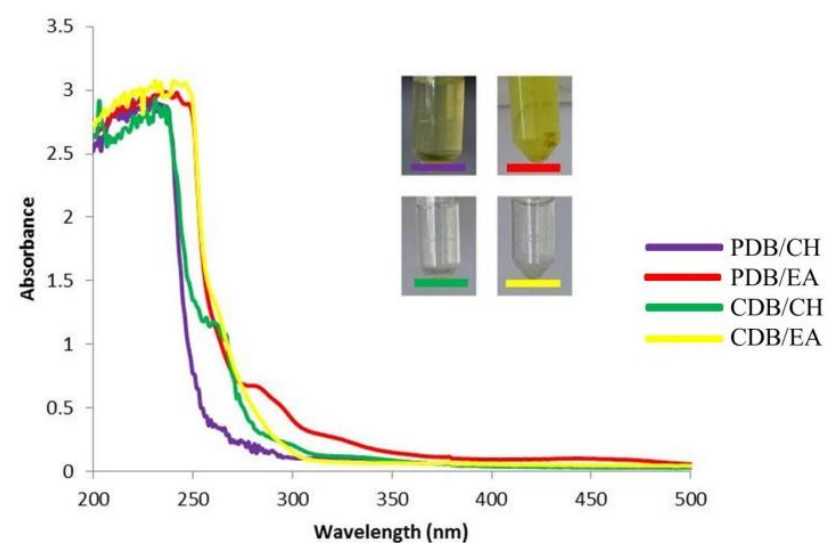

Fig. 9: UV-Visible spectra of culture filtrate of Aspergillus sp. (DUMB 14) grown in different media extracted with different solvents. PDB-Potato dextrose broth; CDB-Czapek-Dox broth; CH-Chloroform; EA- Ethyl acetate. Inset- Solvent extracts used for analysis.

Ethyl acetate extract of Penicillium sp. (DUMB 15) mycelial mass grown on PDB showed intense orange-red pigment and a major peak at $463 \mathrm{~nm}$ (Fig. 10). Yellow, orange and purple pigments were shown to have absorption maxima of $400 \mathrm{~nm}, 470$ $\mathrm{nm}$ and $500 \mathrm{~nm}$ respectively in case of Monascus [23]. Hence, in the present study range of 400 to $500 \mathrm{~nm}$ compounds were detected corresponding to the colour observed in the extract. The culture filtrate did not show such pigments (Fig. 11). However, a significant peak at $235 \mathrm{~nm}$ in culture filtrate from CDB extracted 
in chloroform was not found in other culture filtrates. Hence, this technique may also be used for the chemotaxonomic analysis of fungal metabolites. Spectroscopic analysis of both crude and purified fungal pigments have been reported for qualitative and quantitative detection $[11,18]$.

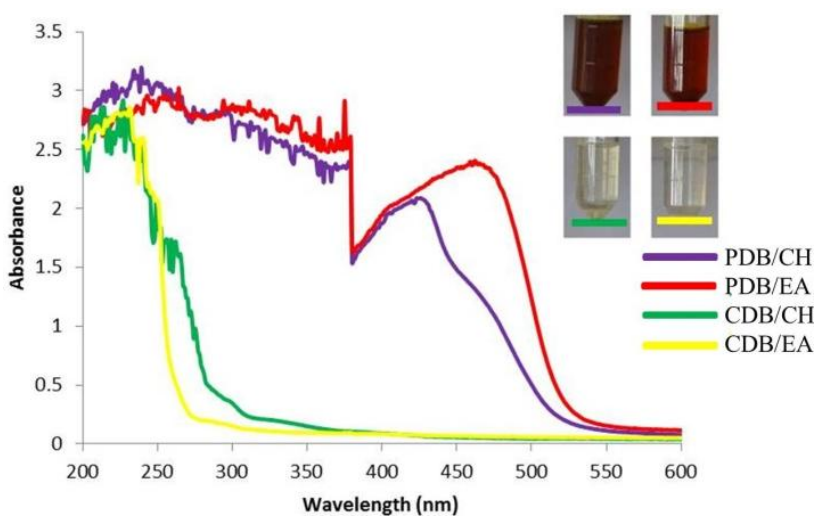

Fig. 10: UV-Visible spectra of mycelial mass of Penicillium sp. (DUMB 15) grown in different media extracted with different solvents. PDB-Potato dextrose broth; CDB-Czapek-Dox broth; $\mathrm{CH}-\mathrm{Chloroform}$; EA- Ethyl acetate. Inset- Solvent extracts used for analysis.

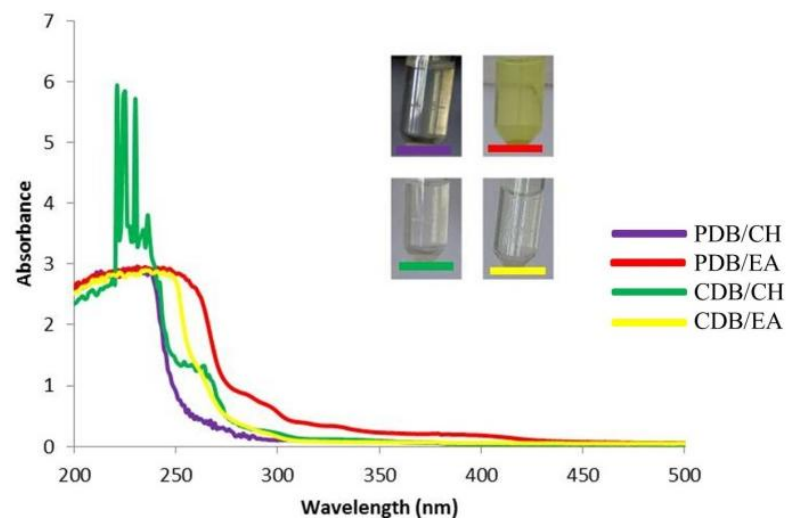

Fig. 11: UV-Visible spectra of culture filtrate of Penicillium sp. (DUMB 15) grown in different media extracted with different solvents. PDB-Potato dextrose broth; CDB-Czapek-Dox broth; $\mathrm{CH}-\mathrm{Chloroform}$; EA- Ethyl acetate. Inset- Solvent extracts used for analysis.

The present study clearly demonstrated production of pigments by fungal strains tested. Both intracellular and extracellular melanin production in Aspergillus nidulans was detected using infra red spectroscopy [9]. Other spectrophotometric techniques are also used for fungal pigment characterization $[10,24,25]$. Hence, it is possible to look for useful pigments not only by visible observation but also by spectroscopy.

\section{CONCLUSION}

In the present study cellular pigments from Aspergillus and Penicillium were detected and spectroscopic confirmation was obtained. It is possible to make use of these fungi for pigment production. Potential of these fungi for biotechnological exploitation and conditions for economically viable protocol needs to be established.

\section{ACKNOWLEDGEMENT}

The authors are grateful to Prof. M. S. Hegde, Convener, Talent Development Centre, Indian Institute of Science, Challakere, Chitradurga for providing laboratory facilities and valuable help for spectral analysis. The spectrophotometric facility provided by Prof. Gopal M. Advirao, Department of Biochemistry is gratefully acknowledged.

\section{Financial support and sponsorship: Nil.}

Conflict of Interests: There are no conflicts of interest.

\section{REFERENCES}

1. Cho YJ, Park JP, Hwang HJ, Kim SW, Choi JW, Yun JW. Production of red pigment by submerged culture of Paecilomyces sinclairii. Letters in Applied Microbiology. 2002; 35:195-202.

2. Joshi VK, Attri D, Bala A, Bhushan S. Microbial pigments. Indian Journal of Biotechnology. 2003; 2:362-369.

3. Celestino JDR, DeCarvalho LE, Lima MP, Lima AM, Ogusku MM, DeSouza JVB. Bioprospecting of amazon soil fungi with the potential for pigment production. Process Biochemistry. 2014; 49:569-575.

4. Kumar A, Vishwakarma HS, Singh J, Dwivedi, S, Kumar M. Microbial pigments: production and their applications in various industries. International Journal of Pharmaceutical, Chemical and Biological Sciences. 2015; 5 (1):203-212.

5. Lian X, Wang C, Guo K. Identification of new red pigments produced by Monascus ruber. Dyes and Pigments. 2007; 73: 121125.

6. Akilandeswari P, Pradeep BV. Exploration of industrially important pigments from soil fungi. Applied Microbiology and Biotechnology. 2016; 100 (4):1631-1643.

7. Torres FAE, Zaccarim BR, Novaes LCL, Jozala AF, Santos CA, Teixeira MFS, Santos-Ebinuma VC. Natural colorants from filamentous fungi. Applied Microbiology and Biotechnology. 2016; 100 (6): 2511-2521.

8. Issac S. Many fungi are brightly colored; does pigmentation provide any advantage to those species? Mycologist. 1994; 8(4):178179.

9. Goncalves RCR, Lisboa HCF, Pombeiro-Sponchiado SR. Characterization of melanin pigment produced by Aspergillus nidulans. World Journal of Microbiology and Biotechnology. 2012; 28:1467-1474

10. Duran N, Teixeira MFS, DeConti R, Esposito E. Ecological-friendly pigments from fungi. Critical Reviews in Food Science and Nutrition. 2002; 42 (1):53-66.

11. Zhong S, Zhang X, Wang Z. Preparation and characterization of yellow Monascus pigments. Separation and Purification Technology. 2015; 150:139-144.

12. Pagano MC, Dhar PP. Fungal pigments: An overview. In: Gupta VK, Mach RL, Sreenivasaprasad S, editors. Fungal biomolecules: Sources, Applications and Recent Developments, USA: John Wiley \& Sons Ltd; 2015, p. 173-181.

13. Vendruscolo F, Buhler RMM, Carvalho JC, Oliveira D, Moritz DE, Schmidell W, Ninow JL. Monascus: a reality on the production and application of microbial pigments. Applied Biochemistry and Biotechnology. 2016; 178:211-223.

14. Caro Y, Venkatachalam M, Lebeau J, Fouillaud M, Dufosse L. Pigments and colorants from filamentous fungi. In: Merillon JM, Ramawat KG, editors. Fungal metabolites. Switzerland: Springer International Publishing; 2015, p. 1-70.

15. Thom C, Raper KB. A manual of the Aspergilli. Baltimore: The Williams and Wilkins company; 1951.

16. Barnett HL, Hunter BB. Illustrated genera of imperfect fungi. 4th ed. USA: Burgess publishing company; 1972. 
17. Chintapenta LK, Rath CC, Maringinti B, Ozbay G. Pigment production from a mangrove Penicillium. African Journal of Biotechnology. 2014; 13 (26):2668-2674.

18. Babitha S, Soccol CR, Pandey A. Effect of stress on growth, pigment production and morphology of Monascus sp. in solid cultures. Journal of Basic Microbiology. 2007; 47:118-126.

19. Yang $\mathrm{H}$, Xiao $\mathrm{C}, \mathrm{Ma} \mathrm{W}, \mathrm{He} \mathrm{G}$. The production of hypocrellin colorants by submerged cultivation of the medicinal fungus Shiraia bambusicola. Dyes and Pigments. 2009; 82:142-146.

20. Pavia DL, Lampman GM, Kriz GS, Vyvyan JR. Spectroscopy. New Delhi: Cengage Learning India Pvt. Ltd. 2007.

21. Ammar MS, Gerber NN, McDaniel LE. New antibiotic pigments related to fusarubin from Fusarium solani (Mart.) Sacc. The Journal of Antibiotics. 1979; XXXII (7): 679-684.

22. Broder CU, Koehler PE. Pigment produced by Monascuspur pureus with regard to quality and quantity. Journal of Food Science.1980; 45:567-569.
23. Martinkova L, Juzlova P, Vesely D. Biological activity of polyketide pigments produced by the fungus Monascus. Journal of Applied Bacteriology.1995; 79:609-616.

24. Huang Z, Zhang S, Xu Y, Li L, Li Y. Structural characterization of two new orange pigments with strong yellow fluorescence. Phytochemistry Letters. 2014; 10:140-144.

25. Mahesh J, Kamat SD, Kamat DV. Fungal pigment characterization and application. Bionano Frontier. 2014; 7(1):34-40.

\section{How to cite this article:}

Narendrababu BN, Shishupala S. Spectrophotometric detection of Pigments from Aspergillus And Penicillium isolates. J App Biol Biotech. 2017; 5 (01): 053-058. DOI: 10.7324/JABB.2017.50109 